\title{
New Useful Heat Flow Equation in Solar Absolute Radiometer and Its Applications
}

\author{
Xiao Tang ${ }^{1,2}$, Ping $\mathrm{Jia}^{2}$, Yupeng Wang ${ }^{2}$, Xin Ye and Wei Fang ${ }^{2, *}$ \\ ${ }^{1}$ University of Chinese Academy of Sciences, Beijing 100049, China \\ ${ }^{2}$ Changchun Institute of Optics, Fine Mechanics and Physics, Chinese Academy of \\ Sciences, Changchun, China \\ *Corresponding author E-mail: fangw@ciomp.ac.cn
}

\begin{abstract}
In order to accommodate with the high accuracy requirement of solar irradiance measurement, a new kind of heat flow equation in solar absolute radiometer is developed in this paper. The detailed solution procedure is present by using the method of separation of variables and principle of superposition. The applications of new equation are also investigated, too. The application in SIAR (Solar Irradiance Absolute Radiometer) shows that the ultimate theoretical value of time constant of SIAR is $18 \mathrm{~s}$ which apparently smaller than the experimental result of $30 \mathrm{~s}$. The result agree with our expectation and helpful for the improvement of SIAR. The application in PMO6 (Physikalisch Meteologisches Observatorium Radiometer-6) shows that the new heat flow equation could deduced from the theory of double exponential function proposed by Gentile, which also proves the correctness of itself.
\end{abstract}

Keywords: solar absolute radiometer, solar irradiance, heat flow equation

\section{Introduction}

Solar absolute radiometer is an electrical substitution radiometer which applied to monitor the variation of solar irradiance [1-3]. The main principle is using electrical substitution method to measure solar irradiance. Electrical power is applied initially to keep the temperature of cavity above that of heat sink in radiometer. When the shutter is opened that radiation falls on the cavity, an electronic feedback system is used to keep the cavity at the same temperature. Therefore, the electrical power reduced is equal to the radiant power which needed to be measured [4-6].

Because of this measurement procedure, it is important to define the appropriate heat flow equation in solar absolute radiometer and predict the dynamic thermal response of the heat transfer system. The existing definition is a zero dimensional heat flow equation which proposed by Fox and Rice in 2005 [7]. It is convenient but also simply for solar irradiance measurement. However, with the development of solar radiometry and increasing requirement of high accuracy of solar absolute radiometer, it is necessary to put forward a new suitable heat flow equation.

In this paper, a new kind of one dimensional heat flow equation is developed to meet the requirement of measurement. In Section 2, we brief review of the heat flow equation proposed by Fox and Rice. In Section 3, we using the new simplified method and obtain the appropriate one dimensional heat flow equation $\Delta T(x, t)$. The applications for solar absolute radiometer are present in Section 4. Finally, the conclusion is present in Section 5

\section{Brief Review of Zero Dimensional Heat Flow Equation}


With the heat diffusion equation and Fourier's laws, and considering a homogeneous medium within which there is no bulk motion, and temperature distribution $T(x, y, z)$ is expressed in Cartesian coordinates, the temperature distribution is given by [8]:

$$
\frac{\partial T}{\partial t}=a\left(\frac{\partial^{2} T}{\partial x^{2}}+\frac{\partial^{2} T}{\partial y^{2}}+\frac{\partial^{2} T}{\partial z^{2}}\right)+\frac{\dot{\Phi}}{\rho c}
$$

where $t, \rho$ and $c$ are the time, density and specific heat, $\dot{\Phi}$ is the rate at which the energy is generated by inner energy source per unit volume of the medium(W/m3), $\alpha=\lambda /(\rho c)$ is the thermal diffusivity, where $\lambda$ is the thermal conductivity of material. Materials of large $\alpha$ will respond quickly to changes in their thermal environment.

A simplified zero dimensional analysis method proposed by Fox and Rice is adopted to simulate this model, and then, the equation (1) is simplified as below:

$$
\frac{\partial T}{\partial t}=\frac{\dot{\Phi}}{\rho c}
$$

while the $\dot{\Phi}$ is consists of two parts:

$$
\dot{\Phi}=\dot{\Phi}_{1}+\dot{\Phi}_{2}=\frac{-K\left(T-T_{0}\right)}{V}+\frac{P_{0}}{V}=\frac{P_{0}-K\left(T-T_{0}\right)}{V}
$$

where $K$ is total thermal conductivity, $T_{0}$ is initial temperature, $P_{0}$ is composition heat power, and $V$ is the volume.

Compiling equation (2) and (3), we obtain:

$$
C \frac{\partial \Delta T}{\partial \mathrm{t}}+K \Delta T=P_{0}
$$

where $C$ is heat capacity, and $\Delta T$ is the temperature difference between heat sink and cavity.

Solving equation (4) with initial condition that temperature difference is zero in the first time. $\Delta T$ is given by:

$$
\Delta T(t)=\frac{P_{0}}{K}\left(1-e^{-\frac{t}{\tau}}\right)
$$

where $\tau=C / K$ is the time constant, it means time consumed to reach $(1-1 / e)$, namely about $63 \%$, times than the maximum temperature difference.

As time $\rightarrow \infty$, temperature difference $\Delta T$ approaches a maximum:

$$
\Delta T_{\max }=\frac{P_{0}}{K}
$$

Therefore, the equation (5) is the given zero dimensional heat flow equation in solar irradiance radiometer. There is no geometrical boundary in zero dimensional modeling, and the spatial position makes no contribute to temperature distribution. The cavity, heat sink and heat links (three main component of solar irradiance radiometer) make up of the heat transfer system. This system is modeled as point elements (i.e. thermal mass) with heat capacity. 


\section{One Dimensional Heat Flow Function}

It is convenient for using the equation (5) to predict the trend of thermal response of solar irradiance radiometer. However, it does not solve the current problem and obtain the enough information which we needed to know.

\subsection{The Transformation of Heat Flow Function}

In this way, we start with the one dimensional heat equation where $x=0$ denotes the position of the heat sink, and $x=L$ ( $L$ is the length of heat link) denotes that of the cavity. To accurate for the temperature variation of heat sink, we apply a time dependent function $f(t)$ at the boundary. With these conditions it is able to formulate the heat flow equation with a known initial value:

Partial deferential equation (PDE) $\frac{\partial T(x, t)}{\partial t}=\alpha \frac{\partial^{2} T(x, t)}{\partial x^{2}}, 0 \leq x \leq L$ and $t \geq 0$

Boundary conditions $(\mathrm{BC})$

$$
\left\{\begin{array}{l}
T(0, t)=f(t), t \geq 0 \\
\frac{\partial T}{\partial x}=\frac{P}{A \lambda_{H L}}, x=L
\end{array}\right.
$$

Initial condition (IC)

$$
T(x, 0)=T_{0}+\frac{P}{A \lambda_{H L}}, 0 \leq x \leq L
$$

We can use the transformation

$$
T(x, t)=U(x, t)+\frac{P x}{A \lambda_{H L}}+f(t)
$$

denotes the thermal conductivity of heat link, and the definition $s(t)=\partial f(t) / \partial t$ to homogenize the boundary conditions:

$$
\begin{aligned}
& \text { PDE } \quad \frac{\partial U(x, t)}{\partial t}-\alpha \frac{\partial^{2} U(x, t)}{\partial x^{2}}=-s(t), 0 \leq x \leq L \\
&\left\{\begin{array}{l}
U(0, t)=0, t \geq 0 \\
\frac{\partial U}{\partial x}=0, x=L
\end{array}\right. \\
& \text { BC } \quad U(x, 0)=0,0 \leq x \leq L
\end{aligned}
$$

Using the transformation $U(x, t)=W(x, t)+Q(x, t)$, where

$$
Q(x, t)=-\frac{s(t)}{2 \alpha} x^{2}+\frac{s(t) L}{\alpha} x_{\text {(i.e. }} Q(0)=0, Q^{\prime}(L)=0, \text { and } a Q_{x x}^{\prime \prime}-s(t)=0 \text { ), }
$$

we can obtain:

$$
\mathrm{PDE} \quad \frac{\partial W(x, t)}{\partial t}=\alpha \frac{\partial^{2} W(x, t)}{\partial x^{2}}, 0 \leq x \leq L
$$




$$
\begin{gathered}
\left\{\begin{array}{l}
W(0, t)=0, t \geq 0 \\
\frac{\partial W}{\partial x}=0, x=L
\end{array}\right. \\
\mathrm{BC} \quad W(x, 0)=-Q(x, 0)=\frac{s(0)}{2 \alpha} x^{2}-\frac{s(0) L}{x} x, 0 \leq x \leq L
\end{gathered}
$$

\subsection{The Solution of $W(x, t)$}

The boundary conditions of $W(x, t)$ accord with the requirement of partial deferential equation can be solved. Therefore, we define $W(x, t)=X(x) T(t)$ and compile with equation (7) to deduce that:

$$
\frac{\partial T^{2}(t)}{\partial t} / \alpha T(t)=\frac{\partial X^{2}(x)}{\partial x} / X(x)
$$

Then, using the method of separation of variables [9], and assume that:

$$
\frac{\partial T^{2}(t)}{\partial t} / \alpha T(t)=\frac{\partial X^{2}(x)}{\partial x} / X(x)=-\beta
$$

It can be deduced that:

$$
\begin{gathered}
\frac{\partial T^{2}(t)}{\partial t}+\alpha \beta T(t)=0 \\
\frac{\partial X^{2}(x)}{\partial x}+\beta X(x)=0
\end{gathered}
$$

Solving equation (18), we can obtain:

$$
T(t)=g e^{-\alpha \beta t}
$$

where $g$ is an arbitrary constant. As compile equation (14) and (19), it can be deduced that:

$$
\begin{gathered}
\frac{\partial X^{2}(x)}{\partial x}+\beta X(X)=0 \\
\left\{\begin{array}{l}
X(0)=0 \\
\frac{\partial X(L)}{\partial x}=0
\end{array}\right.
\end{gathered}
$$

Discussing the cases that (a) $\beta<0$ (b) $\beta=0$; (c) $\beta>0$.

(a) If $\beta<0$, then, the general solution of equation (21) is:

$$
X(x)=A e^{\sqrt{-\beta} x}+B e^{-\sqrt{-\beta} x}
$$

where $A$ and $B$ is arbitrary constants. Solving equation (23) with the boundary conditions from equation (22), it is suggested that the only solution is: $A=B=0$. So, the case (a) $\beta<0$ is wrong.

(b) If $\beta=0$, then, the general solution of equation (21) is: 


$$
X(x)=A+B x
$$

Because $X(0)=0$, it can be deduced that $A=0$; Meanwhile, because $\partial X(L) / \partial x=0$, it can be deduced that $B=0$. Therefore, the case (b) $\beta=0$ is wrong, too.

(c) If $\beta>0$, then, the general solution of equation (21) is:

$$
X(x)=A \cos \sqrt{\beta} x+B \sin \sqrt{\beta} x
$$

Because $X(0)=0$, it can be deduced that $A=0$; Meanwhile, because $\partial X(L) / \partial x=0$, it can be deduced that $B \cos \sqrt{\beta} L=0$. Therefore, only when $B \neq 0$, the equation (21) has untrivial solution.

Because $\cos \sqrt{\beta} L=0 \Rightarrow \sqrt{\beta} L=(2 k+1) / 2 \pi_{(k=0,1,2 \cdots) \text {, it can be deduced that }}$ $\beta_{k}=\left(\frac{2 k+1}{2 L}\right)^{2} \pi^{2}$

, where $\beta$ is consists of a series of $\beta_{k}$. Thus, the solution of equation (18) and (19) are given by:

$$
\begin{aligned}
& X_{k}(x)=B_{k} \sin \frac{2 k+1}{2 L} \pi x,,(k=0,1,2 \cdots) \\
& T_{k}(t)=g_{k} e^{-\alpha\left(\frac{2 k+1}{2 L}\right)^{2} \pi^{2} t},(k=0,1,2 \cdots)
\end{aligned}
$$

so, the variable $W(x, t)$ is given by:

$$
W_{k}(x, t)=X_{k}(x) T_{k}(t)=C_{k} e^{-\alpha\left(\frac{2 k+1}{2}\right)^{2} \pi^{2} t} \sin \frac{2 k+1}{2 L} \pi x
$$

Meanwhile, because of the principle of superposition ${ }^{[9]}$, it can be deduced that:

$$
W(x, t)=\sum_{k=0}^{\infty} W_{k}(x, t)=\sum_{k=0}^{\infty} C_{k} e^{-\alpha\left(\frac{2 k+1}{2}\right)^{2} \pi^{2} t} \sin \frac{2 k+1}{2 L} \pi x \quad,(k=0,1,2 \cdots)
$$

Compiling equation (29) with (15), we obtained that:

$$
W(x, 0)=\frac{S(0)}{2 \alpha} x^{2}-\frac{S(0) L}{\alpha} x=\sum_{k=0}^{\infty} C_{k} \sin \frac{2 k+1}{2 L} \pi x,(k=0,1,2 \cdots)
$$

Assuming that

$$
h(x)=\frac{S(0)}{2 \alpha} x^{2}-\frac{S(0) L}{\alpha} x
$$
then, the equation (30) is equal to Fourier's sine series expansion of $h(x)$. The range of variable $x$ is from 0 to $\mathrm{L}$. However, as calculated, for Fourier's expansion of equation (30), only the first series matters because the second series is reduced nearly another 1 orders of magnitude. Thus, we calculate the case that $W(x, t)=W_{0}(x, t)$ (i.e. $\left.k=0\right)$.

$$
\text { Because } C_{k}=\frac{2}{L} \int_{0}^{L} h(x) \sin \frac{2 k+1}{2 L} \pi x d x,(k=0,1,2 \cdots), \text { so }
$$




$$
\begin{gathered}
C_{0}=\frac{2}{L} \int_{0}^{L} h(x) \sin \frac{\pi x}{2 L} d x \\
=\frac{2 S(0)}{\alpha L} \int_{0}^{L} \frac{x^{2}}{x} \sin \frac{\pi x}{2 L} d x-\int_{0}^{L} x L \sin \frac{\pi x}{2 L} d x \\
=-\frac{16 S(0) L^{2}}{\alpha \pi^{3}}
\end{gathered}
$$

then, we can obtain that:

$$
\begin{aligned}
W(x, t) & =C_{0} e^{-\alpha\left(\frac{\pi}{2 L}\right)^{2} t} \sin \frac{\pi x}{2 L} \\
C_{0} & =-\frac{16 S(0) L^{2}}{\alpha \pi^{3}}
\end{aligned}
$$

\subsection{The New Heat Flow Equation}

As attention before, the variable $U(x, t)$ is given by:

$$
U(x, t)=W(x, t)+Q(x, t)=C_{0} \sin \frac{\pi x}{2 L} e^{-\alpha \frac{\pi^{2}}{4 L^{2}} t}+\frac{S(t) L}{\alpha} x-\frac{S(t)}{2 \alpha} x^{2}
$$

then, we can obtain the one dimensional heat flow equation which we needed as:

$$
\begin{gathered}
T(x, t)=U(x, t)+\frac{P x}{A \lambda_{H L}}+f(t) \\
C_{0} \sin \frac{\pi x}{2 L} e^{-\alpha \frac{\pi^{2}}{4 L^{2}} t}+\frac{S(t) L}{\alpha} x-\frac{S(t)}{2 \alpha} x^{2}+\frac{P x}{A \lambda_{H L}}+f(t)
\end{gathered}
$$

Meanwhile, the temperature of cavity (which denoted as the position of $x=L$ ) is described as:

$$
\begin{gathered}
T(L, t)=C_{0} e^{-\frac{t}{\tau^{\prime}}}+\frac{S(t) L^{2}}{2 \alpha}+\frac{P L}{A \lambda_{H L}}+f(t) \\
\tau^{\prime}=4 L^{2} / \alpha \pi^{2}
\end{gathered}
$$

where $\tau^{\prime}$ is the new time constant of solar irradiance radiometer,

$$
C_{0}=-\frac{16 S(0) L^{2}}{\alpha \pi^{3}}
$$

$\alpha=\lambda / \rho c, S(t)=\partial f(t) / \partial t, f(t)$ is the time function accurate for the temperature variation of heat sink, $P$ is the input power, $A$ is the cross section area of heat link, $\lambda_{H L}$ is the thermal conductivity of heat link, $\lambda, \rho$ and $c$ are the total thermal conductivity, density and heat capacity of heat transfer system, respectively.

\section{Applications}

\subsection{The Application in SIAR (Solar Irradiance Absolute Radiometer)}


The solar absolute radiometer has been developed in Changchun Institute of Optics, Fine Mechanics and Physics (CIOMP) in China since 1990s. In order to establish accurate and stable TSI database, a new package, Solar Irradiance Absolute Radiometer (SIAR), was equipped on the FENGYUN-3A, FENGYUN-3B and FENGYUN-3C satellites launched on May 2008, November 2010 and September 2013, respectively [10-12]. The equation (35) and (36) are used to explain the dynamic thermal response of SIAR.

For example, the time constant is calculated by new definition with equation (36). Learn from the thermal response of cavity from Tang [4], the real time constant of SIAR is approximately 30s. Meanwhile, the theoretical value of time constant calculated by equation (36) is $18.0 \mathrm{~s}$, where the thermophysical properties of materials are taken from the work of Wang et al [13]. The comparison result accommodate with our expectation. Because a kind of expoxy glue is used to agglutinate the heat sink, cavity and heat link together, which obviously increase the time constant of SIAR. The imperfect heat contact between each component also makes concentrate to the enhanced time constant.

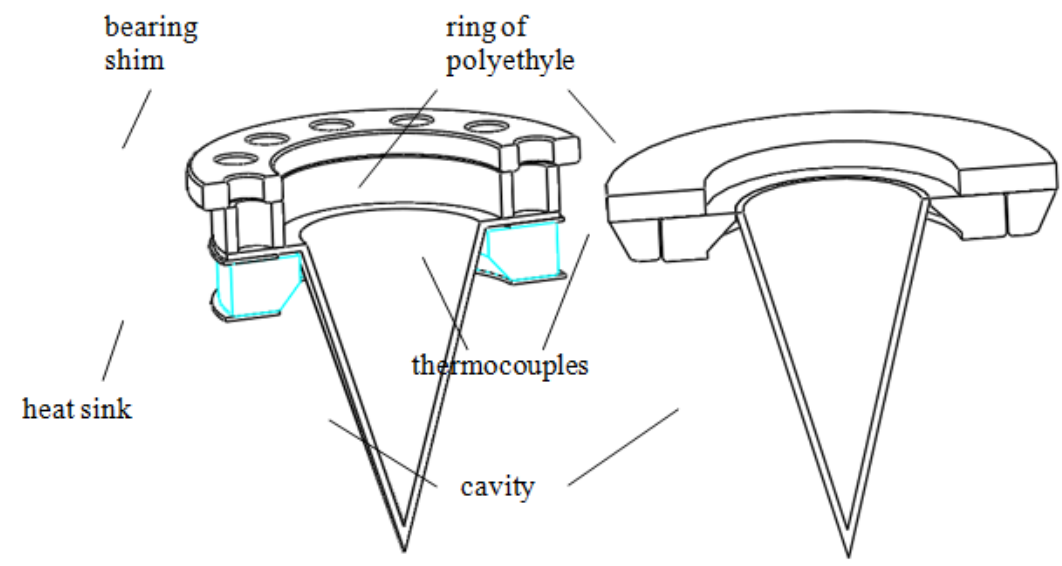

(a)

(b)

Figure 1. (a) New and (b) Previous Configuration of Heat Transfer System of SIAR

Therefore, we design a new configuration of heat transfer system of SIAR to reduce the time constant, as shown in Figure 1(a), where Figure 1 (b) is the heat transfer system of SIAR used before. In Figure 1(a), we add an earlap on the top of cavity, which improve the imperfect thermal contact between thermocouples and cavity. Because the thermocouples are designed to measure the temperature difference between cavity and heat sink by thermoelectric effect, we also change the heat transfer path from cavity to heat sink. It is changed from measure the difference between the left and right surface to top and bottom surface, which enhances the cross section area and advances the heat transfer efficiency. As a result, the new configuration of heat transfer system of SIAR leads to a measured time constant of about 20s, which is more suitable for the measurement of solar irradiance for SIAR.

The equilibrium temperature of cavity is also investigated by this equation, too. As attention before, this temperature can be denoted as

$$
T(L, \infty)=\frac{P L}{A \lambda_{H L}}+\frac{S(\infty) L^{2}}{2 \alpha}+f(\infty)
$$

As time $\rightarrow \infty, f(\infty)$ will be a constant value. Defining that $T^{\prime}=f(\infty)$, obtain $S(\infty)=0$, and then equation (37) is given by: 


$$
T(L, \infty)=\frac{P L}{A \lambda_{H L}}+T^{\prime}
$$

A finite element model ${ }^{[5]}$ is used to calculate the equilibrium temperature of cavity. The discrepancy, which is less than $0.86 \%$, shows that the experimental results and numerical model agree well. Assuming that the temperature of heat sink is maintain at a constant value of $300 \mathrm{~K}$, then the equilibrium temperature of cavity is $300.846 \mathrm{~K}$ in numerical model, and $300.843 \mathrm{~K}$ by calculated with equation (38). The relative error of temperature difference between numerical model and calculated value is less than $0.35 \%$, which proved the correctness of equation (35) and (36).

\subsection{The Application in PMO6 (Physikalisch Meteologisches Observatorium Radiometer-6)}

In order to improve the understanding of thermal characterization of solar absoluter radiometer, Gentile et al in National Institute of Standards and Technology (NIST) have ever developed a radiometer which called High Accuracy Cryogenic Radiometer (HACR) in Gaithersburg [14]. It is suggested that the temporal behavior of $V_{C}$ (the electrical signal of cavity, which can be regard as the temperature of cavity) is described by:

$$
V_{C}=A_{1}+A_{2} e^{-\frac{t}{\tau_{1}}}+A_{3} e^{-\frac{t}{\tau_{2}}}
$$

where $\tau_{1}=116 \pm 3$ s and $\tau_{2}=245 \pm 8$ s. The coefficients $A_{1}, A_{2}$ and $A_{3}$ are determined by linear fitting in real time and closed-form expression. In a word, the temperature of cavity should be described as a function of time to a double exponential function.

However, this function cannot be deduced from the zero dimensional heat flow equation, but, the one dimensional heat flow equation can be the suitable explanation. For example, the solar absoluter radiometer laboratory of PMO6 in Switzerland discovered that the temperature variation of heat sink agrees with a one exponential function as [15]:

$$
f(t)=T_{1}+\Delta S-\Delta S e^{-\frac{t}{\tau_{3}}}
$$

where $T_{1}$ is the initial temperature of heat sink, and $\Delta S$ is a constant value. Combing equation (40) with (35), the temperature of cavity of PMO6 is given by:

In summary, the new heat flow equation perfectly justifies the double exponential function form of temperature of cavity and applicable for all solar absoluter radiometer.

\section{Conclusion}

Accurate measurement of solar irradiance requires meaningful selection of materials. In this paper, a new kind of heat flow equation of solar absolute radiometer is developed to improve the understanding of dynamic response of heat transfer system. It is shows that the new heat flow equation is more convenient and useful for researchers to predict the thermal response of cavity and select the materials. 
The applications of new heat flow equation in solar absolute radiometers are investigated, too. The application in SIAR shows that there is a difference between ultimate value of time constant and that of experimental result, which reveal the way how to improving the configuration of SIAR. Meanwhile, the application in PMO6 shows that this new equation perfectly justifies the double exponential function form proposed by Gentile, which also proves the correctness of itself

\section{Acknowledgement}

This work was supported by the National Natural Science Foundation of China under Grant NO. 41474161 and the National High-tech R\&D program of China (863 Program) under Grand No. 2015AA123703.

\section{References}

[1] B. Mendoza, "Total solar irradiance and climate", Adv. Space Res., vol.35, (2005), pp. 882-890.

[2] G. Kopp, A. Fehlmann, W. Finsterle, D. Harber, K. Heuerman, and R. Wilson, "Total solar irradiance data record accuracy and consistency improvement”, Metrologia, vol.49, (2012), pp. S29-S33.

[3] T. Pulli, T. Donsberg, T. Poikonen, F. Manoocheri, P. Karha and E. Ikonen, "Advantages of white LED lamps and new detector technology in photometry", Light: Science \& Applications vol.4, (2015), pp.2015.105.

[4] X. Tang, W. Fang and Y. Wang, "Effect and Experiment Analysis of First Specular Reflection Error on Absolute Radiometers", Chinese J Lasers, vol.43, (2016), pp. 0408002.

[5] X. Tang, W. Fang, Y. Luo, K Wang and Z. Xia, "Thermal characteristic analysis of heat sink in Absolute Cryogenic Radiometer”, Acta Opt. Sinica., vol.36, (2016), pp. 1012004.

[6] X. Tang, P. Jia, K. Wang, B. Song, W. Fang and Y. Wang, "Non-equivalence correction of solar irradiance absolute radiometer", Opt. Precision Eng., vol.24, (2016), pp. 2370-2376.

[7] N. P. Fox, and J. P. Rice, Editor, "Experimental Methods in the Physical Science", Elsevier Inc., London, (2005).

[8] F. P. Incropera, D. P. Dewitt, T. L. Bergman, and A. S. Lavine, Editor, "Fundamentals of Heat and Mass Transfer (6th edn.)", John wiley \& sons, Hoboken, (2006).

[9] Q. Yang and B. Pu, Editor, "Advanced Heat Transfer (2nd edn.)", Shanghai Jiao Tong University, Shanghai, (2004).

[10] Y. Wang, X. Hu, H. Wang, X. Ye and W. Fang, "Standard transfer chain for radiometric calibration of optical sensing instruments with traceability in solar reflective bands", Opt. Precision Eng. vol.23, (2015), pp. 1807-1812.

[11] D. Yang, W. Fang, X. Ye and B. Song, "Program-controlled sun-tracking precision of spaceborne solar irradiance monitor", Opt. Precision Eng. vol. 23, (2015), pp. 1813-1821.

[12] B. Song, X. Ye, D. Yang, M. Jiang and W. Fang, "Precise measurement of voltages in space cryogenic radiation temperature system”, Opt. Precision Eng., vol.23, (2015), pp. 1903-1910.

[13] W. Fang, H. Wang, H. Li and Y. Wang, "Total Solar Irradiance Monitor for Chinese FY-3A and FY-3B Satellites - Instrument Design”, Solar Physics, vol.289, (2014), pp. 4711-4726.

[14] T. Gentile, J. Houston, J. Hardis, C. Cromer and A. Parr, "National Institute of Standards and Technology high-accuracy cryogenic radiometer", Applied Optics, vol.35, (1996), pp. 1056-1068.

[15] A. Fehlmann, "Metrology of Solar Irradiance (PhD thesis)", Zurich: University of Zurich, (2011). 
International Journal of Multimedia and Ubiquitous Engineering Vol.12, No.4 (2017) 